\title{
Polymorphisms of Homocysteine Metabolism Are Associated with Intracranial Aneurysms
}

\author{
Alexander Semmler ${ }^{a} \quad$ Michael Linnebank $^{a} \quad$ Dietmar Krex $^{c} \quad$ Anika Götz $^{d}$ \\ Susanna Moskau ${ }^{a}$ Andreas Ziegler ${ }^{d}$ Matthias Simon ${ }^{b}$ \\ Departments of a Neurology and ${ }^{b}$ Neurosurgery, University of Bonn, Bonn, 'Department of Neurosurgery, \\ University of Dresden, Dresden, and ${ }^{\mathrm{d}}$ Institute of Medical Biometry und Statistics, University of Lübeck, \\ Lübeck, Germany
}

\section{Key Words}

Homocysteine $\cdot$ Intracranial aneurysm • Polymorphism •

Transcobalamin 2

\begin{abstract}
Background: Impaired homocysteine metabolism is associated with a number of vasculopathies including extracranial aneurysms. We analyzed the possible association of nine genetic variants of homocysteine metabolism with the occurrence of intracranial aneurysms. Methods: Caucasian patients $(n=255)$ treated at two German hospitals for intracranial aneurysms and local controls ( $n=348$ ) were genotyped for the following polymorphisms: methionine synthase (MTR) c. $2756 \mathrm{~A} \rightarrow \mathrm{G}$, methylenetetrahydrofolate reductase (MTHFR) c.677C $\rightarrow$ T, MTHFR c.1298A $\rightarrow$ C, cystathionine $\beta$-synthase (CBS) c.844_855ins68, CBS c.833T $\rightarrow C$, dihydrofolate reductase (DHFR) c.594 + 59del19bp, glutathione S-transferase $\Omega-1$ (GSTO1) c.428C $\rightarrow$ A, reduced folate carrier 1 (RFC1) c.80G $\rightarrow$ A and transcobalamin 2 (Tc2) c.776C $\rightarrow$ G. Results: The G-allele of the missense polymorphism Tc2 c.777C $\rightarrow \mathrm{G}$ was found to be underrepresented in patients, suggesting that this variant may protect from the formation of cerebral aneurysms [odds ratio per two risk alleles (OR) 0.48 ; 95\% confidence interval $(\mathrm{Cl}) 0.30-0.77 ; p=0.002]$. We obtained borderline results for the G-allele of RFC1 c.80G $\rightarrow A$
\end{abstract}

\section{KARGER}

Fax +4161306 1234 E-Mail karger@karger.ch www.karger.com

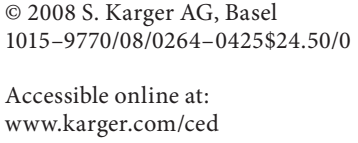

(OR 1.64; 95\% Cl 1.01-2.65; $\mathrm{p}=0.051)$ and the insertion allele of DHFR c.594 + 59del19bp (OR 1.61; 95\% CI 1.00-2.60; $p=$ 0.059), which were found to be overrepresented in patients. Conclusion: Polymorphisms of homocysteine metabolism are possible risk factors for the formation of intracranial aneurysms.

Copyright $\odot 2008$ S. Karger AG, Basel

\section{Introduction}

Aneurysmal subarachnoid hemorrhage caused by ruptured intracranial aneurysms has a 30-day mortality rate of up to $45 \%$, and $30 \%$ of survivors have moderateto-severe disability [1]. Little is known about the etiology of intracranial aneurysms. Hereditary factors are supposed to be involved in the etiology of cerebral aneurysms. In addition to defined diseases like autosomal dominant polycystic kidney disease, multiple genetic susceptibilities are considered to act together in the etiology of subarachnoid hemorrhage [2]. The increase in the familial risk of subarachnoid hemorrhage is approximately fourfold among first-degree relatives [3, 4]. Poly-

\section{A. Semmler and M. Linnebank contributed equally to this work.}


morphisms of genes coding for constituents of vascular walls or involved in angiogenesis and vascular remodeling have been suggested to be associated with the formation of cerebral aneurysms [2]. Polymorphisms affecting homocysteine metabolism have been reported to promote atherosclerosis, ischemic stroke, cervical arterial dissections and abdominal aortic aneurysms [5-7]. In addition, autosomal recessive deficiency in cystathionine$\beta$-synthase (CBS; OMIM 236200), which is involved in homocysteine metabolism, presents concomitant with vascular disease including the formation of aneurysms [8]. The present study aimed to analyze whether polymorphisms affecting homocysteine metabolism are associated with the incidence of intracranial aneurysms.

\section{Patients and Methods}

\section{Subjects}

Patients of Caucasian origin undergoing microsurgery or endovascular treatment for cerebral aneurysms were recruited from the Departments of Neurosurgery at the University of Bonn and the Technical University of Dresden, Germany. Diagnoses were made by brain imaging, including digital subtraction angiography in all cases. Patients with known autosomal dominant polycystic kidney disease, fibromuscular dysplasia or with additional cerebrovascular dysplasia were excluded [9].

This study was approved by the local ethics committees. Written informed consent was obtained from patients or their legal representatives.

As controls we studied 250 apparently healthy Caucasian residents in the area of Bonn (64\% female; mean age $50.4 \pm 11.9$ years) without a history of cerebrovascular disease and 98 Caucasian healthy anonymous blood donors from the Dresden area. The Bonn area residents were recruited from an ongoing study on cerebrovascular disease. They are healthy partners from patients with vascular events, free from any history or ultrasonic signs of vascular disease [10].

\section{Genotyping}

Genomic DNA prepared from peripheral leukocytes was used for genotyping by PCR amplification and restriction analysis of nine genetic variants of homocysteine metabolism including the intronic deletion dihydrofolate reductase (DHFR) c.594+ 59del19bp (affecting the transcriptlevel; GenBankNM_000791.3), the splice alteration cystathionine $\beta$-synthase c.844_855ins68 (affecting the transcript level; GenBank S78267.1) and the missense mutations (i.e. leading to amino acid exchanges) methionine synthase (MTR) c.2756A $\rightarrow$ G (p.D919G; rs1805087), methylenetetrahydrofolate reductase (MTHFR) c.677C $\rightarrow$ T $($ p.A222V; rs1801133) and c.1298A $\rightarrow$ C(p.E429A; rs1801131), CBS c.833T $\rightarrow$ C (p.I278T; rs5742905), glutathione S-transferase $\Omega-1$ (GSTO1) c.428C $\rightarrow$ A (p.A140D; rs4925), reduced folate carrier 1 (RFC1) c. $80 \mathrm{G} \rightarrow \mathrm{A}$ (p.R27H; rs1051266) and transcobalamin 2 (Tc2) c.776C $\rightarrow$ G (p.P259R; rs1801198) [11].

\section{Statistical Analysis}

Deviation from and compatibility with the Hardy-Weinberg equilibrium was analyzed using a Monte Carlo $\chi^{2}$ goodness-of-fit test (C-program available from www.imbs-luebeck.de) [12] and the exact uniformly most powerful equivalence test with $\delta_{2}=2$ and $\delta_{1}=2 / 3$, respectively (SAS macro using SAS version 9.1) [13]. As primary analysis, exact logistic regressions between genotypes and aneurysms were carried out using the Cochran-Armitage trend test for genotypes and stratification by center. Covariates were available for the samples from Bonn, and adjustments for age and gender were performed using the exact logistic regression framework with trend test coding for genotypes (software StatXact 6). Population stratification can be assumed to be negligible in the present study because we extensively investigated population stratification in the entire German population previously [14]. The global test level was set to 5\%. Adjustments for multiple testing were done according to the step-down procedure of ŠidakHolm [15]. Asymptotic 95\% confidence intervals (CIs) were computed for genotypes without adjustments for multiple testing for the joint analysis of both centers. Exact CIs at the nominal 95\% confidence level were calculated for each center without adjustments for covariates (SAS 9.1).

Power calculations using the available sample were carried out using the two-sided asymptotic Cochrane-Armitage test at the global $0.625 \%$ test level that already adjusts for the analysis of eight successfully genotyped polymorphisms assuming the observed odds ratios (ORs) and genotype frequencies in the controls (StatXact 6).

\section{Results}

We investigated 111 patients ( $74 \%$ female, mean age $51.1 \pm 12.0$ years) of Caucasian origin undergoing microsurgery or endovascular treatment for cerebral aneurysms at the Department of Neurosurgery, University of Bonn, and 144 Caucasian patients admitted to the Department of Neurosurgery, Technical University of Dresden (63\% female, mean age $51.1 \pm 12.0$ years). Seven further patients had been excluded due to autosomal dominant polycystic kidney disease $(\mathrm{n}=3)$, fibromuscular dysplasia $(n=1)$ or other cerebrovascular dysplasia $(n=$ 3 ). In $36 \%$ of the cases, more than one intracranial aneurysm was diagnosed.

The GSTO1 c. $428 \mathrm{C} \rightarrow$ A variant showed deviation from the Hardy-Weinberg equilibrium in the controls of Bonn $(\mathrm{p}=0.02)$ and was therefore omitted from further analysis. No other deviations were observed in the control populations.

The genotype distribution of $\mathrm{Tc} 2 \mathrm{c} .777 \mathrm{C} \rightarrow \mathrm{G}$ was significantly different between patients and controls ( $\mathrm{p}=$ 0.002 ; table 1). We observed borderline significant results for DHFR c.594 + 59del19bp ( $\mathrm{p}=0.059)$ and for $\mathrm{RFC1} c .80 \mathrm{G} \rightarrow \mathrm{A}(\mathrm{p}=0.051)$. Analysis of the other poly- 
Table 1. Polymorphisms of homocysteine metabolism and aneurysm formation in Caucasian patients from Bonn $(\mathrm{n}=111)$ and Dresden $(\mathrm{n}=144)$

\begin{tabular}{|c|c|c|c|c|c|c|}
\hline \multirow[t]{2}{*}{ Genotype } & \multicolumn{2}{|c|}{ Patients } & \multicolumn{2}{|c|}{ Controls } & \multicolumn{2}{|l|}{$\mathrm{p}$ value } \\
\hline & $\mathrm{n}$ & $\%$ & $\mathrm{n}$ & $\%$ & OR & $95 \% \mathrm{CI}$ \\
\hline CBS c.833T $\rightarrow$ C: & & & & & $\mathrm{p}=1.00$ & \\
\hline $\mathrm{TT}$ & 250 & 98.8 & 341 & 98.6 & 1.00 & \\
\hline $\mathrm{CT}$ & 3 & 1.2 & 5 & 1.4 & 0.98 & $0.22-4.34$ \\
\hline $\mathrm{CC}$ & 0 & 0.0 & 0 & 0.0 & 0.96 & $0.05-18.8$ \\
\hline CBS c.844ins68: & & & & & $\mathrm{p}=0.440$ & \\
\hline del/del & 216 & 86.7 & 306 & 88.4 & 1.00 & \\
\hline ins/del & 32 & 12.9 & 40 & 11.6 & 1.26 & $0.76-2.08$ \\
\hline ins/ins & 1 & 0.4 & 0 & 0.0 & 1.58 & $0.58-4.33$ \\
\hline MTHFR c.677C $\rightarrow$ T: & & & & & $\mathrm{p}=0.984$ & \\
\hline $\mathrm{CC}$ & 116 & 46.0 & 158 & 45.7 & 1.00 & \\
\hline CT & 109 & 43.3 & 152 & 43.9 & 0.99 & $0.77-1.28$ \\
\hline TT & 27 & 10.7 & 36 & 10.4 & 0.98 & $0.59-1.63$ \\
\hline MTHFR c. $1298 \mathrm{~A} \rightarrow$ C: & & & & & $\mathrm{p}=0.609$ & \\
\hline $\mathrm{AA}$ & 117 & 46.4 & 161 & 46.4 & 1.00 & \\
\hline $\mathrm{AC}$ & 113 & 44.8 & 150 & 43.2 & 0.93 & $0.71-1.20$ \\
\hline $\mathrm{CC}$ & 22 & 8.7 & 36 & 10.4 & 0.86 & $0.51-1.44$ \\
\hline MTR c. $2756 \mathrm{~A} \rightarrow \mathrm{G}:$ & & & & & $p=0.675$ & \\
\hline $\mathrm{AA}$ & 168 & 66.4 & 223 & 64.3 & 1.00 & \\
\hline $\mathrm{AG}$ & 81 & 32.0 & 112 & 32.3 & 0.92 & $0.67-1.27$ \\
\hline GG & 4 & 1.6 & 12 & 3.5 & 0.85 & $0.45-1.61$ \\
\hline DHFR c. 594 + 59del19bp: & & & & & $\mathrm{p}=0.059$ & \\
\hline $\mathrm{del} / \mathrm{del}$ & 35 & 14.1 & 78 & 22.7 & 1.00 & \\
\hline del/ins & 124 & 49.8 & 153 & 44.5 & 1.27 & $1.00-1.61$ \\
\hline ins/ins & 90 & 36.1 & 113 & 32.8 & 1.61 & $1.00-2.60$ \\
\hline RFC1 c. $80 \mathrm{G} \rightarrow \mathrm{A}:$ & & & & & $\mathrm{p}=0.051$ & \\
\hline $\mathrm{AA}$ & 76 & 31.1 & 129 & 38.1 & 1.00 & \\
\hline $\mathrm{AG}$ & 118 & 48.4 & 154 & 45.4 & 1.28 & $1.01-1.63$ \\
\hline GG & 50 & 20.5 & 56 & 16.5 & 1.64 & $1.01-2.65$ \\
\hline $\mathrm{Tc} 2$ c. $776 \mathrm{C} \rightarrow \mathrm{G}:$ & & & & & $\mathrm{p}=0.002$ & \\
\hline $\mathrm{CC}$ & 97 & 38.5 & 96 & 28.1 & 1.00 & \\
\hline CG & 113 & 44.8 & 157 & 45.9 & 0.69 & $0.55-0.88$ \\
\hline GG & 42 & 16.7 & 89 & 26.0 & 0.48 & $0.30-0.77$ \\
\hline
\end{tabular}

Numbers of genotypes (n) observed and ratios (\%) per polymorphism are depicted. Nominal two-sided p values from an exact logistic regression with center as covariate are shown using the trend test coding for genotypes. Asymptotic CIs were calculated for the joint analysis of both centers.

morphisms did not reveal any significant associations.

Although population stratification can be assumed to be negligible in the present study [14], we performed explorative separate analyses for the Bonn and the Dresden populations and found significant differences for the Tc2 variant in the larger Dresden subgroup (patients, CC: 0.43, CG: 0.41, GG: 0.16; controls, CC: 0.23, CG: 0.51, GG: $0.26 ; \mathrm{p}=0.003$ ), but not for the smaller Bonn subgroup, although the GG genotype was underrepresented in both patient populations (patients, CC: 0.33, CG: 0.50, GG:
0.17; controls, CC: 0.30 , CG: 0.44, GG: 0.26; $\mathrm{p}=0.161$ ). Similarly, the distribution of the DHFR genotypes was significantly different between patients and controls in the Dresden population (patients, del/del: 0.14, del/ins: 0.46 , ins/ins: 0.41 ; controls, del/del: 0.27 , del/ins: 0.44 , ins/ ins: $0.29 ; \mathrm{p}=0.010$ ) but not in the Bonn population (patients, del/del: 0.15, del/ins: 0.55, ins/ins: 0.30; controls, del/del: 0.21, del/ins: 0.45, ins/ins: 0.34; $\mathrm{p}=0.809$ ). Concerning the RFC1 polymorphism, the distribution of genotypes was significantly different between patients and controls in the Bonn population (patients, AA: 0.24, AG: 
0.53, GG: 0.23; controls, AA: 0.38, AG: 0.46, GG: 0.16; $\mathrm{p}=$ 0.012 ) but not in the Dresden population (patients, AA: 0.37, AG: 0.44, GG: 0.19; controls, AA: 0.37, AG: 0.45, GG: $0.18 ; \mathrm{p}=0.926$ ).

The power to detect effects of DHFR and RFC1 was only 51 and 52\%, respectively, after adjustments for multiple testing. In contrast, the power to detect the effect of $\mathrm{Tc} 2 \mathrm{c} .777 \mathrm{C} \rightarrow \mathrm{G}$ was $81 \%$ even after adjustments for multiple testing.

None of the polymorphisms investigated was significantly associated with the multiplicity of aneurysms, and no significant association with single or multiple aneurysms was seen in subgroups defined by age (not shown).

\section{Discussion}

We analyzed nine functional polymorphisms of methionine metabolism in a total of 255 German patients of Caucasian origin treated for intracranial aneurysms compared with 348 German controls of Caucasian origin. The distribution of genotypes of Tc2 c.776C $\rightarrow \mathrm{G}$ was significantly different between patients and controls. Additionally, we obtained borderline results for the polymorphismsRFC1 c.80G $\rightarrow$ A andDHFR c.594+59del19bp. Subgroup analysis of the Bonn and the Dresden population only proved a significant effect in one of these populations for each of those three polymorphisms. Although the allele and genotype frequencies did not significantly differ between the two control groups (not shown), we cannot exclude that differences in the selection of controls (healthy partners of patients with vascular disease vs. anonymous blood donors) might have contributed to the different results between the Bonn and the Dresden subgroups. Further, within the limits of any association study, we cannot exclude random errors associated with the small numbers in spite of correction for multiple testing. The results observed in our study require confirmation in a further patient cohort.

Previous studies suggested that the G-allele of the Tc2 c.777C $\rightarrow \mathrm{G}$ polymorphism, which was underrepresented in patients with intracranial aneurysms in our study, affects vitamin $B_{12}$ binding affinity and the ability to transport vitamin $B_{12}$ into tissues [16-18], leading to a reduced remethylation of homocysteine to methionine by vitamin $\mathrm{B}_{12}$-dependent MTR. An impaired homocysteine metabolism may lead to an accumulation of asymmetric dimethylarginine $[19,20]$, which is a major endogenous inhibitor of nitric oxide and is strongly predictive of prema- ture cardiovascular disease and death [21, 22]. It has already been shown that nitric oxide availability is a key requirement for the development of intracranial aneurysms, and intracranial aneurysm formation was prevented by inhibition of nitric oxide synthase (NOS) in a rodent model [23]. The size of intracranial aneurysms is significantly smaller in iNOS-/- mice than in iNOS+/+ mice [24]. eNOS polymorphisms have been investigated for associations with intracranial aneurysm formation and rupture [25]. We therefore speculate that the protective effect of the Tc2 c.776G allele found in the present study might in part be explained by decreased transport capacity of vitamin $B_{12}$ in the blood, leading to increased levels of asymmetric dimethylarginine and thereby more effective NOS inhibition. Also, lower homocysteine remethylation by MTR may lead to a higher homocysteine turnover via CBS and, therefore, to a lower capacity of CBS concerning the second reaction catalyzed by this enzyme, the synthesis of the potent vasodilatator $\mathrm{H}_{2} \mathrm{~S}$, which may contribute to vascular damage [26].

The biochemical consequences of the variant DHFR c.594 + 59del19bp and the RFC1 c.80G $\rightarrow$ A polymorphism are less well defined. DHFR converts dihydrofolate into 5,10-methylenetetrahydrofolate, a methyl group shuttle required for the de novo synthesis of nucleic acids as well as the formation of 5-methyltetrahydrofolate by MTHFR. The 19-bp deletion in intron-1 of DHFR has been associated with the risk of spina bifida [27-29]. It supposedly decreases DHFR gene expression [29], and reduced DHFR expression might plausibly lead to reduced availability of folates for remethylation of homocysteine to methionine. Thus, the DHFR variant might speculatively have similar biochemical effects as the Tc2 variant. The extracellular reduced folate carrier RFC1 takes up reduced folates into the cell [30]. The c.80G $\rightarrow$ A polymorphism has been suggested to affect folate and homocysteine metabolism [31]. The biochemical consequences for intracellular, in particular endothelial, metabolism remain speculative.

In summary, the data of our study suggest that the missense polymorphism Tc2 c.776C $\rightarrow \mathrm{G}$ may be associated with the formation of intracranial aneurysms. In addition, the polymorphisms RFC1 c.80G $\rightarrow$ A and DHFR c. $594+59$ del19bp might also be involved. If these results are confirmed in an independent patient cohort, the biochemical effects of these polymorphisms on the intracranial vasculature should be explored in detail. This may well yield important new insights into the pathogenesis of intracranial aneurysms. 


\section{References}

1 Brisman JL, Song JK, Newell DW: Cerebral aneurysms. N Engl J Med 2006;355:928939.

2 Ruigrok YM, Rinkel GJ, Wijmenga C: Genetics of intracranial aneurysms. Lancet Neurol 2005;4:179-189.

3 Teasdale GM, Wardlaw JM, White PM, Murray $\mathrm{G}$, Teasdale EM, Easton V: The familial risk of subarachnoid haemorrhage. Brain 2005;128:1677-1685.

4 Schievink WI, Schaid DJ, Michels VV, Piepgras DG: Familial aneurysmal subarachnoid hemorrhage: a community-based study. J Neurosurg 1995;83:426-429.

5 Casas JP, Bautista LE, Smeeth L, Sharma P, Hingorani AD: Homocysteine and stroke: evidence on a causal link from Mendelian randomisation. Lancet 2005;365:224-232.

6 Strauss E, Waliszewski K, Gabriel M, Zapalski S, Pawlak AL: Increased risk of the abdominal aortic aneurysm in carriers of the MTHFR 677T allele. J Appl Genet 2003;44: 85-93.

7 Arauz A, Hoyos L, Cantu C, Jara A, Martinez L, Garcia I, Fernandez ML, Alonso E: Mild hyperhomocysteinemia and low folate concentrations as risk factors for cervical arterial dissection. Cerebrovasc Dis 2007;24: 210-214.

8 Yap S, Boers GH, Wilcken B, Wilcken DE, Brenton DP, Lee PJ, Walter JH, Howard PM, Naughten ER: Vascular outcome in patients with homocystinuria due to cystathionine beta-synthase deficiency treated chronically: a multicenter observational study. Arterioscler Thromb Vasc Biol 2001;21:20802085.

9 Simon M, Franke D, Ludwig M, Aliashkevich AF, Koster G, Oldenburg J, Bostrom A, Ziegler A, Schramm J: Association of a polymorphism of the ACVRL1 gene with sporadic arteriovenous malformations of the central nervous system. J Neurosurg 2006;104: 945-949.

10 Moskau S, Golla A, Grothe C, Boes M, Pohl C, Klockgether T: Heritability of carotid artery atherosclerotic lesions: an ultrasound study in 154 families. Stroke 2005;36:5-8.

11 Linnebank M, Moskau S, Farmand S, Fliessbach K, Kolsch H, Bos M, Grothe C, Becker D, Harbrecht U, Pohl C, Wullner U, Klockgether T: Homocysteine and carotid intimamedia thickness in a German population: lack of clinical relevance. Stroke 2006;37: 2840-2842.
12 Ziegler A, König IR: A Statistical Approach to Genetic Epidemiology: Concepts and Applications. Weinheim, Wiley-VCH, 2006.

13 Wellek S: Tests for establishing compatibility of an observed genotype distribution with Hardy-Weinberg equilibrium in the case of a biallelic locus. Biometrics 2004;60:694-703.

14 Steffens M, Lamina C, Illig T, Bettecken T, Vogler R, Entz P, Suk EK, Toliat MR, Klopp N, Caliebe A, Konig IR, Kohler K, Ludemann J, Diaz LA, Fimmers R, Lichtner P, Ziegler A, Wolf A, Krawczak M, Nurnberg P, Hampe J, Schreiber S, Meitinger T, Wichmann HE, Roeder K, Wienker TF, Baur MP: SNP-based analysis of genetic substructure in the German population. Hum Hered 2006;62:2029.

15 Guo W, Romano J: A generalized SidakHolm procedure and control of generalized error rates under independence. Stat Appl Genet Mol Biol 2007;6:article3.

16 Afman LA, Lievers KJ, van der Put NM, Trijbels FJ, Blom HJ: Single nucleotide polymorphisms in the transcobalamin gene: relationship with transcobalamin concentrations and risk for neural tube defects. Eur J Hum Genet 2002;10:433-438.

17 Afman LA, van der Put NM, Thomas CM, Trijbels JM, Blom HJ: Reduced vitamin B12 binding by transcobalamin II increases the risk of neural tube defects. QJM 2001;94: 159-166.

18 Miller JW, Ramos MI, Garrod MG, Flynn MA, Green R: Transcobalamin II 775G $>C$ polymorphism and indices of vitamin B12 status in healthy older adults. Blood 2002; 100:718-720.

19 Ito A, Tsao PS, Adimoolam S, Kimoto M, Ogawa T, Cooke JP: Novel mechanism for endothelial dysfunction: dysregulation of dimethylarginine dimethylaminohydrolase. Circulation 1999;99:3092-3095.

20 Stuhlinger MC, Tsao PS, Her JH, Kimoto M, Balint RF, Cooke JP: Homocysteine impairs the nitric oxide synthase pathway: role of asymmetric dimethylarginine. Circulation 2001;104:2569-2575.

21 Schnabel R, Blankenberg S, Lubos E, Lackner KJ, Rupprecht HJ, Espinola-Klein C, Jachmann N, Post F, Peetz D, Bickel C, Cambien F, Tiret L, Munzel T: Asymmetric dimethylarginine and the risk of cardiovascular events and death in patients with coronary artery disease: results from the AtheroGene Study. Circ Res 2005;97:e53-e59.
22 Cooke JP: Asymmetrical dimethylarginine: the Uber marker? Circulation 2004; 109: 1813-1818.

23 Fukuda S, Hashimoto N, Naritomi H, Nagata I, Nozaki K, Kondo S, Kurino M, Kikuchi $\mathrm{H}$ : Prevention of rat cerebral aneurysm formation by inhibition of nitric oxide synthase. Circulation 2000;101:2532-2538.

24 Sadamasa N, Nozaki K, Hashimoto N: Disruption of gene for inducible nitric oxide synthase reduces progression of cerebral aneurysms. Stroke 2003;34:2980-2984.

25 Krex D, Fortun S, Kuhlisch E, Schackert HK, Schackert G: The role of endothelial nitric oxide synthase (eNOS) genetic variants in European patients with intracranial aneurysms. J Cereb Blood Flow Metab 2006;26: 1250-1255.

26 O'Sullivan SE: What is the significance of vascular hydrogen sulphide (H2S)? $\mathrm{Br} \mathrm{J}$ Pharmacol 2006;149:609-610.

$27 \mathrm{Xu} \mathrm{X}$, Gammon MD, Wetmur JG, Rao M, Gaudet MM, Teitelbaum SL, Britton JA, Neugut AI, Santella RM, Chen J: A functional 19-base pair deletion polymorphism of dihydrofolate reductase (DHFR) and risk of breast cancer in multivitamin users. Am J Clin Nutr 2007;85:1098-1102.

28 Johnson WG, Stenroos ES, Spychala JR, Chatkupt S, Ming SX, Buyske S: New 19 bp deletion polymorphism in intron-1 of dihydrofolate reductase (DHFR): a risk factor for spina bifida acting in mothers during pregnancy? Am J Med Genet A 2004;124:339345

29 Johnson WG, Scholl TO, Spychala JR, Buyske $\mathrm{S}$, Stenroos ES, Chen X: Common dihydrofolate reductase 19-base pair deletion allele: a novel risk factor for preterm delivery. Am J Clin Nutr 2005;81:664-668.

30 Kamen BA, Wang MT, Streckfuss AJ, Peryea $\mathrm{X}$, Anderson RG: Delivery of folates to the cytoplasm of MA104 cells is mediated by a surface membrane receptor that recycles. J Biol Chem 1988;263:13602-13609.

31 Chango A, Emery-Fillon N, de Courcy GP, Lambert D, Pfister M, Rosenblatt DS, Nicolas JP: A polymorphism $(80 \mathrm{G} \rightarrow \mathrm{A})$ in the reduced folate carrier gene and its associations with folate status and homocysteinemia. Mol Genet Metab 2000;70:310-315. 